\title{
NOSTALGIA EN EL CUENTO MINIMALISTA AMERICANO DE FINALES DEL SIGLO XX
}

\author{
Santiago Rodríguez Guerrero-Strachan. Universidad de Valladolid
}

\begin{abstract}
La nostalgia es un elemento recurrente en la literatura norteamericana. El artículo analiza las huellas que deja en la narrativa breve americana de la década de 1980 . Para ello se ha elegido a cuatro autores relevantes: Richard Ford, Raymond Carver, Tobias wolf y Stuart Dybek. El artículo se divide en dos partes. En la primera se analiza la relación que mantiene la nostalgia con los temas de los cuentos y el modo en que influye en ellos. La conclusión es que la nostalgia es un elemento fundamental en los relatos. En la segunda se analiza la estructura narrativa. Las historias se dividen en dos partes, que corresponden en líneas generales al presente y al pasado, aunque se dan excepciones. Subrayan la división en la vida de los personajes y la importancia que el pasado tiene en sus vidas. Por último, hay que concluir que la nostalgia en estos relatos se halla relacionada con las condiciones sociales y políticas de la sociedad contemporánea.
\end{abstract}

Uno de los elementos recurrentes en la literatura americana es la nostalgia. Desde los inicios, aparece como constituyente decisivo del significado de las obras. Resulta cuando menos curioso que un lugar que se descubre y se metaforiza como un nuevo Edén, se defina más por aquello que ya no existe que por lo que está presente. La colonización de los actuales Estados Unidos significó el sometimiento y casi total desaparición de los nativos en nombre de una civilización. El impulso no se detuvo en ellos. Las tesis políticas de Jefferson en lo concerniente al establecimiento de una pequeña república agraria también resultaron derrotadas y dieron lugar a un sentimiento permanente de frustración, pérdida y nostalgia. Notes on the State of Virginia (1785) inaugura de un modo explícito lo que ya latía en la cultura americana en la obra de W. Bradford y otros autores de los primeros tiempos, para quienes el mero descubrimiento del Nuevo Mundo hace que este pierda sus señas de identidad como Edén. De ellos partirá una corriente, la de J. F. Cooper y su retrato de la declinante vida en las praderas de la pentalogía The Leatherstocking Tales (1823 - 1841), H.D. Thoreau, A Week on the Concord and Merrimack Rivers (1849) y Mark Twain, Life on the Mississipi (1883). El monumental empeño narrativo de William Faulkner y de escritores regionalistas, los relatos de George Washington Cable o de Zora Neale Hurston señalan también la pérdida de un mundo y el falseamiento del recuerdo.

Porque la nostalgia es un síntoma de aquello que se ha desvanecido y del lugar que su ausencia revela. Opera entonces la imaginación un movimiento de sustitución de lo perdido 
que tiene como resultado la creación e implantación en el recuerdo de situaciones que nunca se vivieron. En el fondo, y de manera radical, todo afán literario parte de la nostalgia, del recuento de un mundo que en el momento de contarlo es ya pasado, se ha desvanecido, y no queda más remedio que rememorarlo, o lo que es lo mismo, imaginarlo. En la nostalgia el centro se desplaza de lo existente al deseo, "pero sin impulso ni materialidad. Deseo volcado hacia lo que una vez fue y ya no volverá" (Jiménez, 1996: 45), a la fantasía, acaso porque como dijera John Keats: "Pleasure never is at Home". Surge del disgusto por el presente, pero también de las fallas del olvido y las trampas de la memoria. En definitiva es también, según apunta Lesley Speed, "essentially a reflection of the perspective from which the past is viewed" (31). Entraña un recuento y juicio del pasado desde una perspectiva adulta, por lo que la autoridad narrativa es un elemento fundamental de dichos relatos.

De un modo general, enfrentarse a la nostalgia es enfrentarse al Otro que es uno mismo pero imaginado, la imagen de quienes nos creemos. Es también un vivir en el pasado obviamente inventado, convertido en ficción de aquello que fuimos- y un sentir una vaga sensación de acabamiento de una época - presente en las obras de algunos de los escritores arriba citados - y que se refuerza en la nuestra, ahora que el siglo, y con él tantas mitologías, acaba. Por esta simple razón se prefiere en literatura la ausencia a la presencia y el recuerdo a la vivencia. El recuerdo de un pasado mixtificado es un impulso básico de la literatura; al fin, literatura y nostalgia se encuentran unidas más de lo que en un principio imaginamos.

No es de extrañar que el impulso nostálgico permanezca en la literatura americana de finales de siglo. La tradición, una innata tendencia humana y la situación social — el final de una época sin que se vislumbre un recambio adecuado - crean las condiciones culturales apropiadas. Puede parecer en principio insólito que en la narrativa minimalista de los años ochenta, en la órbita de un realismo empeñado en reflejar de manera minuciosa, fiel, y en algunos casos degradada, la realidad americana, hasta el punto a veces de llegar al documento sociológico, rayano en la literatura de ínfima calidad, la nostalgia pueda tener su espacio. En algunos relatos de Raymond Carver, de Richard Ford, de Stuart Dybek y de Tobias Wolff, se configura como el elemento semántico vertebrador. La nostalgia ha merecido la atención de estudios más en el plano de la sicología y la cultura popular (Speed, 1998; Crowell, 1999; Wilson, 1999). En un plano específicamente literario, sin embargo, у а pesar de su importancia, ha sido menos estudiada (Alter, 1993; Fritzman, 1993; Hampsey, 1993). A pesar de su evidente presencia en la literatura americana no ha sido objeto de un estudio global pormenorizado y sólo en contados autores como J.F. Cooper ha atraído la atención repetida de los críticos ${ }^{1}$.

Mi propósito, por tanto, es analizar los relatos "Feathers", "Chef's house", "Gazebo" y "The third thing that killed my father off" de Raymond Carver, "Rock Springs", "Children", "Optimists" y "Communists" de Richard Ford, "Chopin in winter" de Stuart Dybek y "Soldier's joy" de Tobias Wolff en los aspectos temáticos y estructurales que vertebran la nostalgia en el relato minimalista americano de las últimas décadas. Espero demostrar así que la nostalgia es un elemento narrativo que aún perdura en la literatura americana.

1.- Temáticamente, los relatos se presentan, desde un punto de vista general, como el final de una época. Esto, como es natural, admite diversas variantes. La más frecuente es el

$\overline{{ }^{1} \text { En Noviembre de }} 2000$ se celebró un congreso sobre el tema en la universidad francesa de Valenciennes.

Odisea, $n^{\circ} 2,2002$ 
paso de la juventud a la madurez. Así se observa en "Rock Springs", "Children", "Optimists" y "Communists" de Richard Ford, y "Chopin in winter" de Stuart Dybek. El elemento común es el narrador que relata las consecuencias que cierto acontecimiento tuvo en su maduración como persona. No implica únicamente el valor ejemplarizante que los cuentos tienen en los lectores (Benjamin, 1998). Entre los valores más aceptados de la sociedad contemporánea se encuentra el de la juventud (Ago y Levi, 1996; Gil Calvo, 1995; Grossberg, 1992). La permanencia en ella de uno u otro modo es rasgo de esta sociedad. Detrás del deseo por el cual a los cuarenta, e incluso a los cincuenta, una persona sigue siendo joven, late una cierta inmadurez, el miedo a la realidad y la perseverancia en las fantasías de un pasado ya extinguido. Apunta a una falta de adecuación entre la realidad y los deseos, una resistencia a madurar y a integrarnos en la vida adulta, una permanencia en la adolescencia, como paraíso que irremediablemente perdemos. La juventud es, como apunta Erikson: “"a delay of adult commitments' for the purposes of allowing identity formation" (157), identidad que no cuaja pues la permanencia en la juventud se alarga de manera indefinida. Sin duda, el mejor ejemplo de esto último lo encarna John Milner, personaje de American Graffitti (1973) de George Lucas. Milner, a pesar de haber superado con creces la adolescencia, persevera en ella. No abandona la pequeña ciudad para ir a estudiar ni entra dentro del círculo de adultos. Su vida se reduce a errar con su coche en busca de aventuras. Con ello consigue crear su leyenda sin darse cuenta de que está viviendo de manera vicaria un pasado que fue el suyo pero ya no le pertenece. Un vivo sentimiento de nostalgia aureola su figura, y con ella la tristeza por lo que fue, o más bien, por lo que quiso haber sido. El narrador de "Rock Springs", Earl, encarna el prototipo. Se empeña en vivir inmerso en el pasado. En un momento determinado percibe la fragilidad: "It felt like a whole new beginning for us, bad memories left behind and a new horizon to build on. I got so worked up, I had a tattoo done on my arm that said FAMOUS TIMES" (Ford, 3). Tiempos famosos, los buenos tiempos, aquellos que pasaron irremisiblemente. La vida no se detiene; los tiempos cambian y el pasado se mineraliza para convertirse en recuerdo, en nostalgia al fin. No existen ni presente ni futuro en la vida de Earl. La ruptura con Edna le obliga a encarar la vida y a olvidarse de lo que hasta el momento ha sido la vida: los buenos tiempos pasados. Frente a él, Edna, se presenta como la persona que rompe con el pasado: "I can't keep that fantasy going anymore" (23). Edna se decide por una nueva vida, lo que significa romper con lo anterior, con los sueños adolescentes de vagar por el mundo en coches robados, alojándose en pensiones de poca monta, y viajando por carreteras secundarias. "Here I am out here in the desert where I don't know anything, in a stolen car, in a motel room under an assumed name, with no money of my own, a kid that's not mine, and the law after me. And I have a choice to get out of all of it by getting on a bus" (24). En el momento crítico de la ruptura, Ford presenta la nostalgia integrada en el protagonista de la historia; su vida se resume en evocar un pasado del que nunca quiso desprenderse, negándose en todo momento a madurar.

Los relatos restantes de Ford tematizan el paso de la adolescencia a la madurez. En ellos los protagonistas no se encierran en un pasado ilusorio; recuerdan, con un mayor o menor grado de tristeza, los momentos decisivos de su vida. "Children", acaso el más desgarrado por estar únicamente protagonizado por niños, plantea la importancia de la amistad en las relaciones sociales, y los primeros escarceos amorosos en un mundo en el cual lo adulto es una presencia ominosa. En la narración de un día en las vidas de Claude y Lucy, el escritor reproduce a escala reducida el mundo de los adultos. La derrota simbólica que sufre el narrador a manos de Claude, su antagonista, se configura desde un punto de vista 
semántico como el momento decisivo en la historia del narrador. Años depués lo recordará con cariño. Es entonces cuando la añoranza surge: "We were friends. But when you are older, nothing you did when you were young matters at all. I know that now, though I didn't know it then. We were simply young" (98). La última frase señala el retorno sentimental a un tiempo inconsciente, más libre o más hermoso porque ya no existe. El deseo del narrador se desplaza hacia la metáfora que es aquel día. Entonces, como en el momento del recuerdo, se enfrenta a la realidad de una vida sórdida. El pasado impone su presencia, y un cambio semántico sutil reflejado en la última frase: "We were simply young". Simplemente jóvenes, no otra es la disculpa de los errores, los miedos o los fracasos; por lo mismo, es la frase que permite la suplencia del presente por un pasado falseado en el último momento, por una memoria nostálgica. Existe una disculpa y una explicación a lo que un día fue, pero no a lo que es en el momento.

"Optimists" y "Communists" cuentan el tránsito a la madurez desde el punto de vista de un narrador infantil, al igual que "Children". La diferencia radica en que ahora el mundo adulto está presente y la relaciones que se dan en él son la espoleta que desencadenará con el paso del tiempo el recuerdo. Tienen también en común el tema político y las relaciones matrimoniales. Contra tal escenario Ford analiza el enfrentamiento entre presente y pasado y las consecuencias que de él se derivan. "Optimists" es la historia de un fracaso, el del matrimonio de Roy Brinson observado a través de los ojos de su hijo, un adolescente, a quien afectará de manera radical. Las tensiones políticas en el sindicato hacen que Roy esté desquiciado y crea que su mujer tiene un amante. Su comportamiento, como se demostrará más tarde, es exagerado y de funestas consecuencias. Al encontrar a Boyd, el supuesto amante en casa, lo ataca y lo mata de manera involuntaria. Roy termina en la cárcel, su mujer le pide el divorcio, y el sueño de la familia se desvanece para todos ellos. A partir de aquí el narrador crea el mito de los buenos tiempos: "When you're young, these things seem unforgettable and at the heart of everything. But they slide away and are gone when you are not so young" (187). El tiempo ha transcurrido y el narrador se sitúa ya en la perspectiva del hombre maduro. Al inicio del relato lo anuncia: "The year, in other words, when life changed for all of us and forever - ended, really, in a way none of us could ever have imagined in our most brilliant dreams of life" (171). Un halo de melancolía, una sensación de derrota se desprende de las frases. El tema se anuncia en la apertura textual y en el cierre, corroborado por la madre, cuando mucho más tarde vuelve a encontrar a su hijo: "I remember those times very well," my mother said. "They were happy enough times. I guess something was in the air, wasn't there?" (189). El tiempo de la felicidad se ha grabado en el recuerdo de los protagonistas, aureolado por la inevitable mixtificación propia de la memoria.

“Communists", por su parte, relata la relación entre el narrador adolescente, su madre y su novio: Glen Baxter. El niño es testigo del fracaso de la relación, pero aun a pesar de toda la violencia presente, al final del relato confesará: "A light can go out in the heart. All of this happened years ago, but I still can feel how sad and remote the world was to me" (232). Tristeza y lejanía, en el presente así como en el pasado. Una vez más la memoria falsea los datos y los tiñe de una coloración inexistente hasta el momento. Nada parecido se infiere de la lectura previa de los hechos. Al final del relato, y con las reflexiones del narrador como punto de partida, la historia adquirirá una significación distinta. No tanto por el contraste con el presente, que no existe, cuanto por la manera de mirar lo que fue.

"Chopin in winter" de Stuart Dybek tematiza también la pérdida de la infancia y la adolescencia, es decir la pérdida de la inocencia. Los pasos que ha de seguir el protagonista para integrarse en la cultura americana señalan los hitos en dicha pérdida. La construcción

Odisea, $\mathrm{n}^{\mathrm{0}} 2,2002$ 
de su identidad como ciudadano pasa por la integración de las culturas americana y polaca ${ }^{2}$. Al fin, la construcción y la integración se configuran como un rito de paso de la inocencia a la madurez. La diferencia radica en que en el caso de la integración, la nostalgia no es un elemento primario. Por tal razón, no se puede argumentar que el tema principal sea exclusivamente el de la formación de la identidad mediante la cultura. La persistencia de la nostalgia en el relato apunta en un primer momento a la pérdida. Si unimos ambos, surge, y no por casualidad, el problema de la conformación definitiva de la identidad. La cristalización de la personalidad crea en los sujetos un vacío. Al fin y al cabo significa el final de la exploración vital más intensa. A partir de entonces la vida se solidifica y se convierte en un camino previsto y explorado de antemano. En el relato de Dybek, el proceso creativo toma como metáfora básica la música. Gracias a ella, el protagonista adquiere consciencia de la importancia que tiene para él la cultura polaca — no en vano es hijo de emigrantes - y de la imposibilidad de rechazar semejante bagaje cultural. La música de Chopin que interpreta su vecina le reconcilia con su ascendencia familiar. Además la música es también el referente narrativo del lenguaje. Desde el momento en que decide aprender la cultura polaca, nota una mejora en su dominio del lenguaje. Música y lenguaje, por lo tanto, son los factores que narrativamente configuran su proceso de maduración. No es de extrañar entonces que al final la música desaparezca, sea una ausencia, un recuerdo a lo sumo. El silencio se adueña de la madurez. La nostalgia también se encuentra ausente: "Even after I no longer missed her, I could still hear the silence left behind" (161). Una vez que el proceso de maduración ha acabado, todo se ausenta. La vida es un inmenso silencio indiferenciado para el protagonista.

Otra de las variantes temáticas, relacionada con la anterior, es la del final de una época de la vida adulta. En esta subcategoría se encuadran los relatos de Raymond Carver "Feathers", "Chef's house", "Gazebo" y "The third thing that killed my father off". Este último admite también su inclusión dentro del anterior grupo de una manera tangencial. A mi entender, el fin del tiempo es el del padre pero también de manera secundaria es el de su hijo, narrador de la historia. En tal caso, la nostalgia sería la de este último y no la del progenitor. De todos modos, la ambigüedad le sirve a Raymond Carver como medio literario para conectar las experiencias vitales del padre y la del hijo. Sin embargo, el tema dominante es la separación de su padre y Dummy. Como consecuencia de la cría de peces, Dummy se aleja de su amigo hasta el punto de que prefiere a aquellos antes que al padre del narrador. Así lo perciben los otros personajes: "My father wouldn't have anything to do with Dummy after that. Not since Dummy ran him off" (244). Se ha acabado una época. Ante el protagonista ahora se extiende el vacío que surge después de dos decepciones, expresadas en las frases: "The first thing was Pearl Harbor. And the second thing was moving to my grandfather's farm near Wenatchee" (241), y que no puede superar en el caso del tercer fracaso. El presente se desvanece y lo que ocupa el escenario es el recuerdo de aquellos momentos: "Those happy days" (244). Una diferencia lo separa de los demás. El narrador observa la situación, y aunque participa de los hechos, siempre media una cierta distancia entre sus sentimientos y los de su padre. Lo que narra, en consecuencia, no siempre está tranformado por los sentimientos. Hay momentos buenos y momentos malos, aunque predominen los primeros. El significado general del relato no deriva tanto de la recreación de las escenas

\footnotetext{
${ }^{2}$ Así lo expuse en el Primer Congreso de Literatura Norteamericana Contemporánea celebrado en Huelva en noviembre de 1999. La ponencia se titulaba "Creación de una identidad mediante la cultura: los relatos de Stuart Dybek", y que está en prensa con el título "Creating an identity by means of culture: Stuart Dybek's short stories".
} 
cuanto de los comentarios del narrador, que casi siempre se centran en la idea de los buenos tiempos pasados: "Those happy days" y "it was like so long to good times and hello to bad" (250). Junto con la nostalgia, la conclusión nos revela un elemento que siempre la acompaña: la idea de final de una época. No hay nostalgia sin dicho sentimiento. Sólo cuando se cierra un tiempo y es imposible recuperarlo, cuando ya no nos pertenece e ingresa en el terreno del deseo y de la fantasía, es cuando la nostalgia aparece.

No es casualidad que los tres primeros reflejen el mismo interés y que se relacionen con algunos de los relatos de Richard Ford. El fin de una etapa está representado por la ruptura del matrimonio. "Feathers" contrapone una noche en casa de un matrimonio amigo escena de tintes grotescos y degradados - con el presente narrativo. Ayuda un comentario del narrador-protagonista de la historia a marcar el significado: "That night at Bud and Olla's was special" (Carver, 306). El contraste entre dos realidades desagradables no es suficiente. Se necesita el paso del tiempo, o lo que es lo mismo, el ingreso en el imperio del recuerdo, para que una de las dos se desvirtúe y, por lo mismo, se sitúe en un plano superior. El contraste es mayor por la propia estructura del relato. Al descubrirse al final la intención de Carver, la sorpresa se acentúa. De un relato que en un principio quiere mostrar simplemente la sociedad americana media, el autor pasa al tratamiento de un conflicto íntimo, el del paso del tiempo y los arañazos que asesta a las personas.

El tema dominante en "Chef's house" es el del recuerdo de un tiempo pasado. Así lo declara la narradora en la primera frase: "That summer Wes rented a furnished house north of Eureka" (308). En un principio puede parecer una frase aséptica, pero el uso del demostrativo la marca afectivamente. El uso de "That" no tiene como función señalar el tiempo pasado. Al reducir este a un momento muy determinado, aparece un matiz significativo que lo remite al ámbito de la nostalgia. A ello ayudan otras frases posteriores: "I want you to try and be the Wes I used to know. The old Wes. The Wes I married" (308). El deseo de la narradora es el de vivir en el pasado. Rechaza un presente que le es adverso y se sitúa en un tiempo que para ella fue mejor. Así lo dice: "I caught myself talking like it was something that had happened in the past" (310). En su mente efectúa una operación mental por la cual elimina la realidad del presente para trasladarse al pasado. Se vive de manera vicaria un tiempo que no llegó a existir. No es cierto que el pasado fuera mejor; no al menos en el caso del cuento analizado. Por el contrario, es un tiempo ocupado por el alcoholismo de Wes, por la ruptura familiar y por la huida y el intento de reconstruir una vida. Es el fracaso de esto último lo que lleva a la protagonista a desear - y por lo tanto a falsear - un tiempo pretérito. Hasta el punto de que no le importa cambiar lo que en el momento de relatarlo son ellos: "Suppose, just suppose, nothing had ever happened. Suppose this was for the first time. (...) Say none of the other had ever happened" (311). La apuesta supera lo corriente. No se trata ya de mitificar una realidad. Se trata de abolirla; de borrarla en su totalidad del tiempo. Se crea así un espacio en blanco que se puede rellenar con la vivencia de un falso recuerdo; es decir con el propósito de la narración. La narradora relata el intento frustrado de reconstruir, o simplemente de crear, un pasado desde el presente. El deseo se derrumba porque se ven obligados a regresar a la realidad, es decir, tienen que abandonar la casa que alquilaron y en la que ella creyó que podría crear un mundo de ensueño gracias a sus deseos.

El último de los relatos de Raymond Carver que trata el tema del fracaso del matrimonio, y con él el final de un ciclo y la revelación de una realidad más amarga, es "Gazebo". La mujer, Holly, se entera de que el marido le ha sido infiel. Esta circunstancia provoca en ella un cambio que se anuncia como el fin. Ese es el sentido que tiene la conversación de los

Odisea, $n^{\circ} 2,2002$ 
protagonistas en la habitación (199-200) y el comentario del narrador: "We both knew it then. We'd reached the end of something, and the thing was to find out where new to start" (202). La conciencia de que un tiempo se ha acabado es necesaria, pero no suficiente. La nostalgia requiere también del recuerdo de los buenos tiempos. Así aparece en la segunda parte del relato: “' 'When we were just kids before we married?' Holly goes. 'When we had big plans and hopes? You remember?" (202). Holly se refiere al tiempo dominado por el recuerdo y el deseo; por tanto, el tiempo de la falsificación. Ante una situación de tensión, ante la incertidumbre de lo por venir, la tendencia es a refugiarse en lo ya conocido, en un tiempo vivido y que no guarda sorpresas. El narrador es consciente de las trampas que elabora la memoria y de que los tiempos no son mejores ni peores sino que el tiempo los dulcifica: "Holly, these things [la crisis matrimonial], we'll look back on them too" (203). Cuando el tiempo pase, el tiempo de la crisis no parecerá tan grave, la habrán asimilado y, por ello, la preferirán a la perplejidad del presente.

Las relaciones familiares se configuran como uno de los elementos claves dentro de los relatos minimalistas (Wolff, 1994: xv). La familia es el microcosmos en el que se reproducen los comportamientos de la sociedad. En vez de explicaciones de amplio calado social, los escritores prefieren el ejemplo pequeño de una sociedad a escala. De ahí que las rupturas familiares de Carver o de Richard Ford no se reduzcan a fijar los momentos decisivos en la vida de una persona; metaforizan rupturas de la sociedad. El recuerdo de lo que fue es reflejo de la parálisis de una sociedad, la ausencia de futuro narrativo lo es del vacío que encara la sociedad contemporánea (King, 1994). Detrás del decorado triunfalista y egocéntrico de la sociedad americana de los años ochenta, se ocultaban síntomas y semillas de una profunda insatisfacción que se veía obligada a expresarse metafórica o analógicamente.

"Soldier's joy" de Tobias Wolff se ocupa de un tema recurrente en la literatura, y en especial en el cine, de los Estados Unidos en la década de los setenta y de los ochenta. La guerra de Vietnam significó un impacto tremendo en las conciencias de los americanos (Snowman y Bradbury, 340 - 341). Sobresale en el relato la añoranza de un tiempo que el lector sabe horroroso. Comparado con el presente del soldado Hooper, se alza como maravilloso. “'My best time,' Hooper said. (...) 'Vietnam,' he said. (...) 'We didn’t know it then,' Hooper said. '(...) But ever since then it's been nothing but confusion'” (Wolff, 1985: 112-113). Los malos momentos se transforman con el paso del tiempo en algo que se añora porque la distancia impone un orden y elimina la incertidumbre. El protagonista echa de menos aquello de lo que carece en el presente. Vive en una situación humillante, degradado en sus responsabilidades y sin que nadie lo tenga en cuenta. La nostalgia es un sentimiento reactivo contra el presente. Así lo expone el narrador: "He tried to bring it back for Porchoff (...) the beauty of that life, the faith so deep that in time you were not separate men anymore, but part of each other" (113). Una líneas más arriba el mismo narrador ha dado ya la clave. Cuando se refiere al mejor momento de la vida comenta: "For Hooper it was closer than the memory of home. In truth it was a kind of home. It was where he went to be back with his friends again, and his old self" (113). Son las trampas de la memoria las que construyen un espacio hogareño, un lugar seguro donde el yo se encuentre reconciliado consigo mismo y con la sociedad, en el que no exista separación entre el individuo y la sociedad, así como tampoco entre la realidad y el deseo. También representa una incapacidad del protagonista para enfrentarse con el presente desagradable en que se halla inmerso.

2.- Estructuralmente, el esquema general es el de la contraposición entre dos tiempos: el pasado y el presente. Lo más frecuente es que el relato se inicie con la narración de los 
hechos pretéritos y sólo al final el narrador se sitúe en el presente. Así son los relatos de Richard Ford "Children", "Optimists" y Communists", y los de Raymond Carver "Feathers" y "Chef's house". Esta disposición narrativa de los hechos es la que mejor subraya la nostalgia. El marcado contraste entre las dos épocas pone de relieve lo que hay de pérdida en el paso de una a la otra, y el estado inferior del presente. Es también propio de este esquema que el cierre textual tome la forma de una revelación. Frente al tiempo indiferenciado en el que se ha vivido, el protagonista - la mayoría de las veces el mismo narrador - acepta la realidad en la que tiene que vivir. Los relatos de Ford son paradigma de lo expuesto. No es sólo la añoranza del tiempo pasado lo que tiene frente a sí. El comentario final sirve como cierre de la narración enlazando el inicio con el presente. John Gerlach denomina a este cierre textual como encapsulación (1985: 12). Es un cierre textual que tiene evidentes coincidencias con el de las películas de adolescentes, American Graffiti entre ellas (Speed, 29-30). Así es en "Children". El comentario del narrador: "We were friends. But when you are older, nothing you did when you were young matters at all. I know that now, though I didn't know it then. We were simply young" (Ford, 98), enlaza los dos tiempos de la narración. De este modo se señala la ausencia de todo aquello que no sea aquel tiempo. Lo mismo puede decirse de "Optimists". El encuentro final del narrador y de su madre conecta ambos momentos. Está reforzado por el inicio: "All of this that I am about to tell happened when I was only fifteen years old" (171). La primera frase anuncia ya el tipo de relato con que el lector se va a encontrar. Las últimas frases exponen además la idea de que nada hay que no sea aquello. La madre se despide: "[she] held me for a moment that seemed like a long time before she turned away, finally, and left me there alone" (191). La madre pertenece al pasado. Es una presencia que sólo aparece en el tiempo en que fueron felices. El regreso es sólo una interrupción en el proceso normal de la vida, un breve espejismo que no tarda en desvanecerse. Los buenos tiempos pertenecen al pasado. Pueden volver por un breve tiempo llamados por el deseo; pero al final, son siempre la soledad, el vacío, el recuerdo los que mueven a escribirlo. "Communists" de una manera oblicua cierra el hueco temporal: "And how old was I then? Sixteen. Sixteen is young, but it can also be a grown man. I am forty-one years old now, and I think about that time without regret, though my mother and I never talked in that way again, and I have not heard her voice now in a long, long time" (235). No hay presente, nada más que el recuerdo de lo que fue y el vacío de lo que no es ya, la ausencia de la madre, de la presencia del pasado por lo tanto. Por su parte "Chef's house" está semánticamente marcado desde el inicio, como ya he señalado: "That summer Wes rented a furnished house..." (Carver, 308). El final se proyecta hacia la nada: "That will be the end of it" (312). Ante el final del sueño, la narradora se enfrenta a un tiempo que no existe, el futuro. La vida fuera de la casa no tiene sentido, o lo que es lo mismo no existe. La aparición de la voz del narrador al final del relato confiere una autoridad narrativa al mismo. La historia se convierte en una revisión de un tiempo pasado desde el punto de vista de la experiencia. Lo normal es que sea de tipo conservador; sin embargo, en el caso de Richard Ford lo único que se aprecia es la ausencia de futuro y la falta de sentido del presente. No supone, por lo tanto, una enmienda a quien entonces fue, sino una comparación en la que el presente sale perdiendo pues el narrador, ya adulto, se percata de que no hay nada y de que sólo le quedan las ilusiones de entonces.

"Feathers" no anuncia en ningún momento del desarrollo narrativo la conclusión. Es el relato en que los dos momentos están separados de manera más nítida. Sólo los comentarios del narrador en la segunda parte lo cierran sobre sí mismo, uniendo ambos momentos. Carver resuelve "Feathers", ya lo he indicado, con un giro sorprendente. En la última parte

Odisea, $\mathrm{n}^{\circ} 2,2002$ 
del relato el narrador protagonista recuerda la velada. La división es, como en historias anteriores, en dos partes, la primera de las cuales es el pasado y la segunda, el presente. Lo diferencia del resto la ausencia de comentario narratorial al inicio, de tal modo que el lector sólo al final se percatará del tipo de relato al que se enfrenta. Por la misma razón en un primer momento parece que su tema es el de la crítica social de la clase media norteamericana; tema, por otro lado, omnipresente en el autor.

Ocurren, sin embargo, excepciones a la regla. La más importante es la inclusión del pasado dentro del presente, de tal modo que a lo sumo, aquel forma una mínima parte de la historia. El ejemplo es "Soldier's Joy". No hay un momento pasado que el narrador escenifique en la historia. Se trata más bien de la mención del protagonista de aquel entonces. El pasado sólo acude en la forma del recuerdo mencionado; está ausente de la estructura narrativa. Para los protagonistas no existe más que el tiempo actual de sus vidas, lo otro es memoria, deseo por tanto, y mitificación. También "Gazebo" se aparta de la estructura general. El pasado aparece simplemente mencionado al inicio de la segunda parte: "'When we were just kids before we married?' Holly goes. 'When we had big plans and hopes? You remember?" (202). Apenas unas líneas imprimen a la historia un rumbo diferente al esperado. Situarlas allí no es casual. El comentario de Holly resta importancia a la primera parte. La separación se ve de ese modo reforzada. El relato se estructura en un primer momento introductorio cuya única función es la de dar paso a la segunda parte, el verdadero corazón de la historia. Por tal razón, el primer momento es difícil de entender. Todo se sobreentiende: el lugar, el tiempo, los hechos. Es necesario avanzar en la lectura para comprender la propuesta narrativa. Las menciones al adulterio anuncian lo que vendrá a continuación. La constatación en la primera parte de que ha finalizado una etapa da lugar al recuerdo en la segunda. El esquema se ha invertido: primero confirman la ruptura para a continuación recordar los tiempos que fueron. Los comentarios finales del protagonista añaden un significado suplementario. Aceptar que cuando el presente se haya convertido en pasado adquirirá las características de cualquier tiempo ya vivido, es admitir un mecanismo propio de las personas por el cual la memoria adapta el recuerdo al deseo; es decir, a la ausencia.

"Rock Springs" incluye el tiempo pasado dentro del presente. El relato no se divide en dos secuencias temporales diferenciadas. No existe más que el presente. Tampoco hay analepsis narrativas que interrumpan el discurso, ni siquiera referencias a aquel tiempo. El pasado se manifiesta en el tatuaje: "Famous Times", recuerdo palpable de entonces y del tipo de vida de Earl. Su vagabundeo por una vida marginal es el recuerdo de tiempos pasados, algo que Edna quiere abolir. En realidad, el relato sólo presenta el pasado mientras que el presente narrativo no pasa de ser una secuela. El presente comienza en el momento en que Edna sube al autobús y abandona a Earl. Edna es de todos los protagonistas la que más se parece a los amigos de John Milner. El sentimiento de haber traspasado la frontera de la adolescencia la embarga. Se da cuenta de que sus vidas reflejan la falsedad de un tiempo clausurado. La falta de adecuación entre lo que son y lo que viven es palpable en Edna. Viven en el pasado desde el presente, y ello le causa el sentimiento de un tiempo perdido.

"The third thing that killed my father off" centra la atención en el pasado. El presente es el del narrador y, no por casualidad, no aparece en el relato a excepción de la primera frase del relato: "I'll tell you what did my father in" (Carver, 241). La frase no se refiere, por lo demás a las acciones narrativas sino al mismo hecho de narrar; afecta nada más que al acto de enunciar y al contenido. El final, al igual que en la mayoría de los casos de Richard Ford, es una reflexión del narrador acerca de lo vivido. Lo diferencia que en el caso de Carver no hay

Odisea, $\mathrm{n}^{\circ} 2,2002$ 
una referencia a la temporalidad interna; es decir, el lector intuye que las consideraciones del narrador no se sitúan en el pasado, pero sin embargo no puede anclarlas textualmente en el presente. Este se ha ausentado de la historia. A lo sumo mantiene una presencia fantasmal y en cierto modo amenazadora, porque rubrica la pérdida del tiempo de la felicidad.

"Chopin in winter" también se caracteriza por presentar simplemente el tiempo pasado. El narrador relata su infancia. El marco narrativo que forman la primera frase del relato y el último párrafo señala el tipo de narración. "The winter Dzia-Dzia came to live with us in Mrs. Kubiac's building on the Eighteenth Street was the winter that Mrs. Kubiac's daughter, Marcy came home pregnant from college in New York" (141). La referencia temporal se dirige a un tiempo en cierta medida mitificado, ausente del curso normal de la vida. El narrador recuerda dos hechos que van a determinar su vida. La estancia de su abuelo con ellos y el regreso de Marcy, la pianista. La consecuencia es su fascinación, gracias a sendos personajes, por la cultura polaca. El párrafo final es un comentario desde el presente del narrador. Al igual que en la historia anterior, el lector no sabe con exactitud desde qué presente está dicho. Imagina que es desde la madurez del narrador, pero no puede asegurarlo con total certeza. El presente narrativo no se encuentra en la narración. Dybek añade un nivel más al dejar ausente también el sentimiento de nostalgia. El protagonista de "Chopin in winter" va más allá de los otros protagonistas al enfrentarse a un tiempo en el que incluso el deseo y la mitificación de lo acontecido está ausente del relato, tanto temática como estructuralmente.

La división en dos partes bien definidas tiene como propósito marcar las dos fases del proceso y separarlas de manera nítida para que el lector sea consciente de la imposibilidad de regreso que late. Se subrayan de este modo los problemas que encaran los personajes para enfrentarse al presente, pues aunque viven en la segunda etapa, anímicamente no han abandonado la primera. Además, la aparición de la voz del narrador en la coda implica una revisión del pasado que, en la mayoría de los casos, suele ser de signo conservador o incluso de negación absoluta del pasado.

La nostalgia en los cuentos que nos ocupan aparece asociada a los momentos en que la fantasía y el deseo se hacen presentes. Como ya he señalado, sin embargo, este es un deseo que no tiene un objeto en el que volcarse sino que lo es de algo que ha desaparecido o de algo que no ha llegado a acontecer. Lo más común es el rechazo del presente que se ve suplantado por un pretérito producto de la imaginación del personaje, y cuya expresión más acertada es la frase de "los buenos tiempos pasados". Late en el fondo la imposibilidad de asumir los retos del presente. Los escritores minimalistas americanos no lo plantean de una manera abiertamente política, sino que se centran en los problemas que los personajes tienen en su vida familiar. El círculo de acción se encuentra reducido a lo que denominamos vida privada. La nostalgia se centra en la añoranza por la pérdida de un tiempo feliz, cuya verdadera calificación es un tiempo más inconsciente. Casi todos los relatos tematizan el paso de la inocencia a la madurez, tema que podría también extrapolarse a la esfera pública, la política más en concreto. F. Jameson ha señalado el papel destacado de la nostalgia en Postmodernism, or the Cultural Logic of Late Capitalism (279-96). La nostalgia supone el mantenimiento de conductas de tiempos pasados. En el fondo, no es más que el síntoma de la incapacidad para abrir nuevos caminos en las vidas, privadas o sociales, de las personas. Si bien, Jameson arguye que la razón última se encuentra en el agotamiento del Capitalismo, no es menos cierto que dicho acabamiento tiene lugar en una época eminentemente conservadora como es la década de los años 80, en la cual tanto Ronald Reagan en América como Margaret Thatcher en Gran Bretaña iniciaron una política conservadora que habría de

Odisea, $n^{\circ} 2,2002$ 
afectar profundamente a sus respectivos países y por ende al resto del mundo. Los escritores de esos años se vieron ante una tesitura histórica, que si bien ya venía siendo anunciada por algunas señales, desde entonces se manifestó aquella con total claridad. Que el nombre que haya recibido sea el de Postmodernidad es en el fondo lo de menos. El sufijo indica la ruptura con la continuidad histórica, que para mí se remonta a la Revolución Francesa y en un estadio algo más embriónico al Renacimiento, y la implantación de un nuevo ciclo histórico con una lógica diferente. En el terreno artístico, y más en concreto en el literario, domina una revisión imitativa de los modelos de períodos anteriores. Es decir, que el realismo o el vanguardismo que se puedan apreciar en dichas obras, no surgen de un impulso realista o vanguardista tal y como hasta ahora los conocíamos, ni tampoco es producto de la ironía moderna que imperó entre los siglos XIX y XX. El elemento definitorio en las Artes de finales del siglo XX es el pastiche; y este es consecuencia de la ausencia de sentido estético en la obra artística. Con ello no quiero decir que la literatura del período carezca de calidad - discusión que más bien saldría de los límites del trabajo —; antes bien señalo una falta de innovación que en términos generales había latido en la literatura hasta finales de 1960.

Por lo mismo, la nostalgia de los relatos analizados, si bien puede asimilarse temáticamente a la que está presente en obras anteriores en la literatura americana, es sin embargo cualitativamente diferente. No subyace el sentimiento de pérdida de una época o de un paisaje, ni siquiera de desaparición de un modo de vida. Los escritores reflejan el paso de la adolescencia a la edad adulta, que bien puede interpretarse como de la inocencia al desengaño. Reflejan en los cuentos no una situación histórica centrada en un número reducido de personajes, sino que crean mínimas situaciones privadas de vidas carentes de heró́smo o interés, y que por ello se refugian en el recuerdo de momentos que los protagonistas creen gloriosos o importantes o al menos merecedores del recuerdo.

No creo oportuno esbozar conclusiones de mayor alcance en lo que se refiere al conjunto de las obras de los escritores, pues con la excepción de R. Carver, los demás están en una fase aún temprana de su carrera literaria, lo que significa que aún pueden añadir matices nuevos a lo ya hecho, y que invalidarían las conclusiones de sesgo general que aquí se pudieran hacer. Ahora bien, sí creo que no es la nostalgia la que lleva al pastiche y a los temas de la vida familiar. El sentido es el contrario; el interés que manifiestan por tales temas y la estética postmodernista que los anima les lleva a que en determinadas ocasiones fijen su atención en la nostalgia como tema gracias al cual pueden exponer una visión de la vida y de la sociedad contemporánea.

\section{BIBLIOGRAFÍA}

Ago, R. y L. Giovanni, dir. 1996. Historia de los Jóvenes. 1, De la Antigüedad a la Edad Moderna. Madrid: Taurus.

Alter, R. 1993. "Modernism and nostalgia". Partisan Review 60, 3: 388-402.

Benjamin, W. 1998. "El narrador". Para una Crítica de la Violencia y Otros Ensayos. Iluminaciones $I V$. Madrid: Taurus.

Carver, R. 1985. The Stories of Raymond Carver. Will You Please Be Quiet, Please? What We Talk About When We Talk About Love. Cathedral. Londres: Picador. 
Crowell, S. 1999. "Spectral history: narrative, nostalgia, and the theme of the I", Research in Phenomenology 29: 82-104.

Dybek, S. 1994. "Chopin in winter". The Vintage Book of Contemporary American Short Stories. Ed. T. Wolff. Nueva York: Vintage.

Erikson, E. H. 1974. Identity: Youth and Crisis. Londres: Faber and Faber.

Fritzman, J.M. 1993. "The future of nostalgia and the time of the sublime". CLIO 23, 2 : 167-189.

Ford, R. 1988. Rock Springs. Nueva York: Vintage.

Gil Calvo, E. 1995. Prisa por Tardar. Madrid: Taurus.

Grossberg, L. 1992. We Gotta Get Out of this Place: Popular Conservatism and Postmodern Culture. Nueva York: Routledge.

Hampsey, J. C. 1993. "Houses of the mind: the architecture of the childhood". The Antioch Review 51, 3: 251-263.

Jameson, F. 1991. Postmodernism, or the Cultural Logic of Late Capitalism. Londres: Verso.

Jiménez, J. 1996. Memoria. Madrid: Tecnos.

King, R. 1994. "The eighties". Introduction to American Studies. Eds. M. Bradbury y H. Temperley. Londres: Longman. 362 - 381.

Snowman, D. y M. Bradbury. 1994. "The sixties and seventies". Introduction to American Studies. Eds. M. Bradbury y H. Temperley. Londres: Longman. 323 - 361.

Speed, L. 1998. "Tuesday's gone: the nostalgic teen film". Journal of Popular Film and Television 26, 1: 24-32.

Wilson, J.L. 1999.“'Remember when...' A consideration of the concept of nostalgia”. ETC 56, 3: 296-304.

Wolff, T. 1994. "Introduction". The Vintage Book of Contemporary American Short Stories. Ed. T. Wolff. Nueva York: Vintage. xi-xvi. 1996. Back in the World. Nueva York: Vintage. 